\title{
La question de la citation : entre la pratique littéraire et la pratique juridique
}

Laté LAWSON-HELLU, Université Western

Citation. «Passage cité d'un auteur, d'un personnage célèbre et donné comme tel (généralement pour illustrer ou appuyer ce que l'on avance). »

(Le Petit Robert 1. 1993 : 383)

\section{La problématique}

Pour la perspective théorique postcoloniale, l'œuvre littéraire postcoloniale, c'est-à-dire produite dans un contexte de colonisation et trouvant son intelligibilité dans ce contexte du point de vue de l'individu colonisé, n'est pas uniquement le lieu d'expression d'une individualité, mais aussi et surtout celui de l'expression du rapport de l'individu au collectif soumis au fait colonial. Par la résistance se comprend ainsi l'outil de lecture proposé par une telle perspective herméneutique qui situe la pertinence sémiotique de l'œuvre littéraire dans son fondement discursif et politique. Pour Jean-Marc Moura, la perspective postcoloniale naît notamment d'un « sens politique de la critique littéraire » et, comme proposé ici, elle inscrit toute la pratique littéraire, celle de l'écrivain comme celle du critique universitaire, dans la perspective juridique qui informe également le fait littéraire, l'un des aspects de cette perspective juridique étant le recours à la citation. Moura définit la perspective postcoloniale :

D'abord mouvement critique, puisqu'il s'agissait de décrire, d'interpréter et d'évaluer les effets qu'un ensemble inédit d'œuvres en langues europhones pouvait avoir sur les lecteurs, les études postcoloniales se sont rapidement orientées vers une histoire littéraire visant à déterminer les conditions de production, puis de réception des textes. Les deux ont mené à des théories, développant des points de vue métacritiques, à des réflexions sur les présupposés de cette critique et de cette histoire comme sur les modalités de production du sens ou de la valeur qui les caractérisent. $[\ldots]$

La perspective postcoloniale n'est pas purement littéraire [...], elle naît d'un sens politique de la critique littéraire, la préoccupation politique n'étant pas une alternative aux préoccupations morales des belles lettres, mais ces préoccupations morales elles-mêmes considérées dans toutes leurs implications, y compris celles qui relient les valeurs d'un individu aux conditions matérielles de son existence. (67) 
C'est, par exemple, dans le roman d'Ahmadou Kourouma, En attendant le vote des bêtes sauvages, que nous évoquerons les termes politiques de la perspective postcoloniale telle qu'en fait état la réflexion de Moura. En cela, nous rappellerons trois cas de rencontre entre la fiction et son référent extratextuel dans ce seul roman de l'écrivain. Il s'agit d'abord de l'histoire politique du «pays du Maghreb avec un roi », comme l'indique l'écrivain, ou « Pays des Djebels », dans sa guerre d'indépendance contre les « Français » :

C'était à l'époque où les Français, déjà vaincus et chassés d'Indochine, s'accrochaient à un autre pays d'Afrique du Nord. Ils n'avaient pas tout à fait oublié la guerre des Djebels après la guerre 14-18, ils se rappelaient la bravoure légendaire des guerriers des Djebels. Ils ne pouvaient pas s'offrir, tenir un autre front en Afrique du Nord. Ils n'eurent pas le choix. Ils allèrent chercher le roi exilé pour que les habitants des Djebels cessent de chercher son effigie dans le ciel et d'entendre sa voix dans les cimetières pendant les nuits noires. Les Français lâchèrent le Pays des Djebels, lui donnèrent son indépendance avec la précipitation de la bête qui crache la boule brûlante qu'il avait imprudemment happée. (261)

Il s'agit également de l'histoire du « dictateur au totem chacal » de ce pays, et de sa mise au service d'intérêts étrangers dans les cadres géostratégiques de la Guerre froide :

Ah ! Koyaga. Vous avez achevé votre visite initiatique par un pays musulman du Nord de l'Afrique, le pays du potentat au totem chacal du désert, les Pays des Djebels et du Sable. Cet autocrate avait la charge, pendant la guerre froide, de mater toutes les rébellions qui se présentaient en Afrique au moyen de parachutistes qu'il dépêchait par des Transalls (les avions de transport de troupes) que la grande République du Nord méditerranéen généreusement mettait à sa disposition. (256-257)

Nous prendrons enfin comme troisième cas, l'histoire de la résistance du Vietnam telle que célébrée dans le roman :

Ah ! Tiécoura, les Vietnamiens sont les Pygmées d'Asie, de frêles Pygmées. Ils ont chassé de leurs terres tous les grands peuples de l'univers. Peuples grands par le nombre de leurs habitants comme les Chinois ; peuples grands par les moyens techniques de leur armée comme les Américains ; peuples grands par leur culture et leur histoire comme les Français. Il est à parier que, si l'univers entier s'alliait pour occuper le sol vietnamien, les Viets vaincraient et jetteraient les soldats du monde entier à la mer. (32-33)

Parmi les quatre conditions proposées par Barbara Harlow pour distinguer le récit de résistance, figure la nécessité d'ancrage du récit symbolique, fictif, dans un territoire et une histoire reconnaissables par le lecteur, lui permettant de retrouver la démarche politique qui informe la mise en récit de l'histoire. Pour Harlow, pour le rappeler ainsi, le « récit de résistance » (80-85) 
suppose en effet la présence d'acteurs appropriés, l'écrivain et les sujets collectifs concernés par l'écriture, lesquels, pour le contexte colonial, deviennent ceux de l'espace colonisé ; la présence d'un objectif approprié, ou la mise en scène et l'interrogation du caractère problématique de l'espace concerné par l'écriture, c'est-à-dire, pour le contexte colonial, l'interrogation de l'histoire coloniale de l'espace colonisé ; la présence d'un contexte spécifique, en l'occurrence l'espace de référence implicite mais évident de l'écriture, lequel, pour le contexte colonial, demeure l'espace culturel et social problématisé par le fait colonial ; la présence de moyen approprié, ou encore la valeur représentative des formes et contenus de l'écriture dans le questionnement de l'histoire problématisée de l'espace de référence, ce qui, pour le contexte colonial, devient la valeur représentative des formes et contenus de l'écriture dans le questionnement de l'histoire coloniale. Les passages d'histoire qui émaillent le roman de Kourouma, rendent compte de ces diverses conditions d'identification du « récit de résistance ».

Il en va ainsi, également, pour prendre ce deuxième exemple, du passage rappelant l'histoire coloniale de la naissance du Togo, actuel territoire national en Afrique de l'Ouest, dans le prologue d'un des romans de Félix Couchoro, L’héritage, cette peste (1963). Rappelons que l'écrivain, né en 1990 au Dahomey, actuel Bénin, et décédé aux lendemains des indépendances africaines en 1968 au Togo, fait partie des écrivains de la première génération littéraire francophone en Afrique subsaharienne. Il fait l'objet de la dernière partie de la réflexion présentée ici. Le passage évoqué de son roman n'inscrit pas seulement ce rappel historique en symbolisme discursif pour l'œuvre, mais y souligne le vice de forme juridique de l'acte juridique ayant porté la constitution officielle de la colonie allemande devant devenir le Togo actuel, la signature du traité de protectorat :

Dans un précis d'histoire du Togo, nous lisons ces quelques lignes qui auréolent de gloire la vieille cité de Baguidah :

Début juillet 1884.

Quand le Mowe jette l'ancre au large d'Anecho, les commerçants allemands viennent à bord. On leur avait donné huit jours pour quitter le territoire. Ils sont contents de voir Nachtigal et sa canonnière. Le consul général descend à terre, palabrer avec le roi d'Anecho. Sans succès. Nous sommes au 3 juillet 1884.

Le Mowe lève l'ancre et remonte la côte, emmenant les commerçants. $A$ Baguidah, le lendemain, Nachtigal fait plonger une lance dans le sol, lance portant l'étendard impérial. Le 5, Nachtigal signe avec Mlapa III, Roi de Togo (aujourd'hui Togoville) un traité mettant les sujets de Mlapa " sous protection allemande ».

Cela, c'est 1'histoire. $(1,327)$ 
$\mathrm{Si}$, dans ce passage, le rappel historique souligne l'invalidité de l'acte juridique, du fait de l'absence du signataire qui devait en valider la représentativité juridique, notamment le roi d'Anecho, il revient à l'analyse critique d'en faire état et, ce faisant, de produire une lecture du roman avec des incidences qui invalident jusqu'à l'existence de l'actuel État issu de cet acte juridique, l'État togolais. Le rapport historique proposé par Peter Sebald sur les circonstances entourant cet acte juridique fait état également de ce vice de forme juridique sans pourtant le nommer, comme le reprend Couchoro en ouverture à son roman. Pour Sebald, en effet :

C'est tout à fait logiquement que le Consul général, envoyé par l'Empire pour fonder des colonies en Afrique de l'Ouest, le Dr. Nachtigal, débarqua le 2 juillet 1884 tout d'abord à Little Popo [Anecho]. C'est parce qu'il ne pouvait pas signer à Little Popo un accord de protectorat avec le "Roi" Lawson qui entretenait de bonnes relations avec la Grande Bretagne que Dr. Nachtigal revint à Bag[u]ida à bord de la "Mouette", et signa là-bas, le 5 juillet, un accord de protectorat avec un groupe de chefs originaires de Togo(ville). C'est ainsi que le nom "Togo" fit son apparition dans l'histoire internationale. [...]

Après que la colonie togolaise eut été administrée un an durant par l'agent commercial Heinrich Randad (1855-1938) nommé consul auparavant, le juriste Ernst Falkenthal (1858-1911), consul impérial, débarqua sur la côte togolaise le 26/6/1885 en compagnie du sous-officier Bilke. Il établit sa résidence et son siège à Baguida, où le traité avait été signé en 1884 avec les chefs de Togo(ville). Il se rendait parfaitement compte que Baguida ne pouvait être qu'une solution provisoire, car, à l'issue des négociations menées par les puissances coloniales, soit la colonie serait cédée entièrement à une autre puissance coloniale, soit elle serait agrandie. Falkenthal avait à peine le moyen d'influencer les résultats des négociations, mais il avait devant lui 17 mois pour déterminer l'endroit où le siège définitif de l'administration devait être fixé.

Cette longue période s'explique par le fait que "petit Popo" était devenu de droit une possession allemande au terme du traité du 24/12/1885, mais qu'il a fallu attendre pratiquement un an pour que Petit Popo soit soumis de facto à l'administration allemande et qu'on y hisse le drapeau allemand le 17/11/1886. (240-241)

Les implications juridiques d'une lecture qui réinscrit ici le contenu de la fiction dans sa pertinence discursive, sociale et politique, c'est-à-dire dans l'intentionnalité de l'écrivain, sont énormes et forment la base du travail herméneutique de la perspective postcoloniale.

La réflexion présentée ici sur la pratique de la citation s'inscrit dans une problématique qui touche de près la pratique de la recherche universitaire en soi en tant que pratique intellectuelle, mais aussi en tant que pratique juridique, comme en témoigne la notion du plagiat qui lui est concomitante dans son intelligibilité négative et judiciaire. 
$\mathrm{Si}$, en effet, la pratique de la citation constitue une part importante de la diffusion des résultats de la recherche universitaire, il s'agit de revenir d'un point de vue épistémologique sur la place de la citation dans l'intelligibilité même de la recherche universitaire pour en souligner la portée juridique et, partant, lever l'équivoque qui, à notre avis, l'entoure, car entre le volume de la citation et sa valeur assertive, le consensus actuel semble porter davantage sur le volume, qu'il est alors généralement recommandé de réduire au minimum. La présentation de ce consensus dans son caractère problématique permet de resituer la pratique de la citation dans sa dimension argumentative foncière, assertive et juridique notamment, c'est-à-dire dans son cadre épistémologique où le principe du volume cède le pas à celui de l'évidence qui fonde sa valeur juridique ou assertive. La réflexion est suivie d'un cas d'étude qui démontre l'invalidité de la consigne minimaliste quant au recours à la citation.

\section{La question de la citation}

Ce sont les termes du consensus sur le volume minimal de la citation que reprend par exemple l'auteur du manuel Initiation pratique à la méthodologie des sciences humaines (Angers), ne serait-ce qu'à partir de sa rubrique consacrée à la question méthodologique dans les sciences humaines et, particulièrement, aux trois méthodes retenues d'ordinaire dans ce domaine du savoir, du moins en ce qui concerne le ministère de l'Enseignement Supérieur et de la Science du Québec des années 1980. Dans ces trois méthodes que sont la méthode expérimentale, la méthode historique et la méthode d'enquête, c'est la méthode historique, dans sa présentation, qui touche au domaine littéraire et, particulièrement, à la problématique de la citation soulevée ici.

Pour l'auteur du manuel, la méthode historique vise la « reconstitution du passé par un examen des événements passés à partir principalement de documents et d'archives » (64) et, en cela, implique la mise à disposition de divers documents, puis leur évaluation ou critique. Cette dernière étape, pour le manuel, comporte deux volets que l'auteur identifie à la critique externe et à la critique interne. Si la critique externe, dite aussi d' « authenticité » ou d' « érudition », consiste «à retrouver l'origine d'un document, c'est-à-dire à le dater et à identifier son ou ses auteurs et son lieu d'origine, ainsi qu'à évaluer son état, c'est-à-dire à constater s'il est entier ou non, altéré ou non, déchiffrable ou non », la critique interne traite de la majeure partie du travail du critique littéraire universitaire. Pour l'auteur du manuel, la critique interne, dite aussi d' « interprétation », ou de « crédibilité », « consiste [ainsi] à vérifier la signification véritable du contenu d'un 
document » (64), et c'est à ce niveau qu'intervient la nécessité de l'authentification des documents devant servir de base à l'étude entreprise. Dans ce cadre, intervient notamment le contexte de production du document :

On se penche alors sur le contenu et sur les raisons de sa production. On examine ce qui y est dit, dans quelle intention, dans quel contexte plus large ces propos s'inscrivent et ce qu'ils pouvaient signifier pour les contemporains de l'époque. On se demande aussi si les événements relatés l'ont été également par d'autres auteurs, s'il n'y a pas de contradictions dans les propos tenus et si l'auteur a été témoin de ce qu'il rapporte et, sinon, sur quelles informations il s'appuie. De cette manière, il devient plus difficile de se méprendre sur le sens et la portée d'un document lors de son utilisation éventuelle dans une analyse d'une période historique donnée. (64)

C'est dans le chapitre portant la conclusion du manuel et consacré au rapport de recherche, qu'apparaît le consensus sur la minimalisation de la citation, ou de ce qui constitue les « appuis au texte » dans le compte rendu de la recherche, autrement dit, de la publication de ces recherches :

Les tableaux, diagrammes, figures, citations, notes d'observation sont les appuis au texte, en ce sens qu'ils soutiennent les propos écrits, mais ne les remplacent d'aucune façon. Par exemple, on peut dégager un tas de constatations de tout ordre d'un tableau parce qu'il ne fournit pas lui-même une direction pour le lire. C'est le chercheur ou la chercheuse, à la lumière de son hypothèse, qui en dégage les éléments essentiels à retenir. Le tableau lui sert par conséquent de support à la démonstration. C'est dans le même sens qu'on présente, par exemple, une citation d'une entrevue, une note d'observation, un extrait d'un document ou une définition statistique. Cela prouve d'une autre façon que c'est d'abord la réflexion, l'analyse, exprimée en mots et en phrases, qui prime dans un rapport de recherche en sciences humaines, et l'abondance de figures ou de tableaux, par exemple, n'est pas un signe en soi d'un travail plus scientifique. (343 ; passage souligné dans le manuel)

Une telle conclusion empiète sur la nécessité d'objectivité évoquée dans les exigences de la méthode historique, par exemple, et se méprend sur la valeur assertive de l'appui au texte, ou de la citation, pour nous, alors que cette valeur est évoquée jusque dans les indications relatives au rapport de recherche, en l'occurrence, le passage relatif aux appuis au texte qui «soutiennent les propos écrits » (343).

Dans sa réalité, la citation, dans la recherche littéraire, constitue de fait un argument d'assertion d'une réflexion proposée à partir d'une œuvre littéraire - son commentaire, par exemple - et à partir de réflexions existantes - thèses, monographies, articles scientifiques, par exemple. Forte de cette réalité, elle modifie les termes d'usage qu'elle infère, du plagiat à l'emprunt, ou à la notion d'auteur qui renvoie aussi bien à l'écrivain qu'au critique universitaire. 
Ainsi, le plagiat, en tant qu'emprunt non mentionné, devient applicable autant à l'auteur qu'au critique universitaire. De même, le critique universitaire, de son statut usuel et présupposé de simple commentateur d'une œuvre, devient plutôt un producteur de savoir à partir de cette œuvre, par exemple, d'où le principe du droit d'auteur appliqué au texte publié de ce critique. La notion d'auteur, c'est-à-dire de propriétaire intellectuel de l'œuvre produite du point de vue du savoir qui est y proposé, renvoie comme d'habitude à l'écrivain, mais aussi au critique universitaire dont le travail vise à produire un nouveau savoir par le biais des modalités du texte d'opinion, du texte argumentatif ou de l'essai, en référence à un corpus donné, dans bien des cas. En cela, la définition que Marilyn Randall propose de l'auteur dans sa réflexion sur la problématique du plagiat renvoie autant à l'écrivain qu'au critique universitaire :

Un écrivain devient donc auteur, si, premièrement, il est le possesseur légitime d'un savoir. Ce droit lui revient du fait qu'il a une connaissance directe des auteurs anciens, lus dans les textes originaux. Deuxièmement, il est préférable que l'écrivain soit l'unique possesseur de ce savoir, selon la règle qui veut que la rareté d'une marchandise augmente sa valeur : l'écrivain accède au rang d'auteur en devenant à son tour objet de citation. Le véritable espace de l'autorité et de l'auteur se situe, pour Bayle, dans les marges du texte, lieu autrefois privilégié du commentateur.

La distinction entre auteur, critique et plagiaire parait fragile : le plagiaire peut être en fait un commentateur qui essaie de se faire passer pour un auteur. C'est que la distinction entre critique et plagiaire dépend moins du fait d'une imitation textuelle que de la légitimité des droits de propriété exercés sur le savoir par l'écrivain. C'est dire, en même temps, qu'un « auteur » ne saurait être plagiaire, étant par définition le propriétaire légitime de son savoir. (94-95)

La citation, comme pratique, participe ainsi du principe de l'argumentation qui fonde le travail du critique universitaire et qui, par ce biais, confère à sa propre argumentation une dimension assertive, c'est-à-dire une dimension juridique, car visant à authentifier son travail de critique. À l'exemple de Babacar Faye, critique universitaire francophone, qui, dans « Noncoïncidence du dire et mise en scène de l'hétérolinguisme dans les écritures francophones africaines », confère à la citation la dimension d'une altérité constitutive du discours proposé par le critique qui en fait usage, et dans le sens où cette altérité mérite sa part d'intégrité et non son assujettissement à celle du discours proposé, la longueur de la citation devient relative face à sa fonctionnalité argumentative. La citation participe notamment du principe de la parenthèse, dont il propose, dans le cadre de sa propre réflexion sur la non-coïncidence du dire ${ }^{1}$, les définitions fournies par le dictionnaire : 
Dans le Nouveau Petit Robert, on peut lire par rapport à la parenthèse : «Insertion, dans le corps d'une phrase, d'un élément qui, à la différence de l'incise, interrompt la construction syntaxique ; cet élément. (...). Phrase ou épisode accessoire dans un discours. » Et dans le Grand Larousse de la langue française : «Phrase, membre de phrase ou groupe de phrases complètement indépendant grammaticalement de la phrase principale, et qui, inséré dans cette phrase ou placé à la fin, en précisent, en rectifient ou en atténuent le sens. (...). Développement accessoire, digression. » Mettre entre parenthèses consiste donc en une opération de décrochement d'un segment discursif pour ramifier son discours, y revenir pour indiquer des instructions de lecture lorsque l'on soupçonne une certaine noncoïncidence du dire. (145-165)

Ici, la «quantité » ou la « longueur » de la citation, son volume, en somme, devient secondaire par rapport à l'argumentation qu'elle vient appuyer, et ne saurait tomber dans le cadre du plagiat, par exemple, que si ses sources ne sont pas signalées.

La problématique de la citation relève donc moins de la question de la copie ou de l'emprunt, comme dans le cadre du plagiat, où il est souvent évoqué, que de la dimension assertive d'une pratique par laquelle le critique recourt à une source externe pour appuyer une argumentation donnée, laquelle peut être le commentaire sur une œuvre donnée, ou la présentation d'une problématique spécifique dans laquelle s'insère par exemple cette œuvre. En cela, le travail du critique universitaire diffère de celui de la critique journalistique, dont l'équivalent universitaire demeure le compte rendu de lecture ou les «notes de lecture » publiées dans les revues. Là, il s'agit de «présenter » une œuvre donnée. Pour la recherche universitaire, c'est bien plutôt d'une question de production de savoir qu'il s'agit, où le critique demeure le propriétaire intellectuel du savoir produit, c'est-à-dire un auteur, en marge de l'œuvre dont un écrivain constitue également, et pour sa part, le propriétaire intellectuel, ou l'auteur. On comprend, dans ce sens, la critique universitaire dont les résultats de recherche, lorsque publiés, deviennent des «œuvres », des essais, des monographies, etc., assorties de droits d'auteurs.

La conception assertive de la citation trouve son application dans le domaine juridique où elle remplit la même fonction argumentative que dans le cadre littéraire évoqué ici, ou, pour le dire dans le sens du discours du droit, sa fonction participative à la prise de décision judiciaire. Il en est ainsi de la réflexion qu'en propose Frédéric Géa, juriste français :

L'épistémologie monologique du droit s'enferme, de par ses présupposés, dans des cadres de pensée qui ne permettent pas d'accéder à l'intelligence du droit, dans toute sa complexité. Réduite à présenter l'acte interprétatif comme un acte neutre, objectif, consistant pour l'interprète à découvrir le sens (supposément) 
préexistant du ou des textes interprétés, sans participer activement à sa détermination, ou, au contraire, comme l'exercice d'un pouvoir discrétionnaire, cette conception reste prisonnière de la dichotomie radicale entre connaissance et volonté. Astreinte à choisir l'un des termes de cette opposition, elle se montre en définitive incapable d'appréhender la dualité caractéristique du processus interprétatif - entre connaissance et volonté, justement. Lui échappe, à ce titre, cette manière de mise en dialogue par laquelle l'interprète, en particulier le juge, s'efforce, en tant que tiers, de cerner les rouages, le montage, la signification, du dispositif textuel, dans un mouvement de « compréhension responsive » [...], avant de codéterminer le sens du texte, grâce à la position d'extériorité qui est la sienne. (277)

Si la théorie du droit ne dispose pas d'un concept qui lui permettrait d'exprimer cette intervention du processus interprétatif dans les prises de décision judiciaires, c'est dans la réflexion de Mikhaïl Bakhtine qu'émerge ce concept qu'il identifie à l'exotopie. Pour Géa, qui applique alors le paradigme de l'exotopie dans sa réflexion visant à réhabiliter au discours du droit sa dimension dialogique, à l'encontre de sa perception monologique d'usage, la prise de décision judiciaire repose non sur l'immuabilité présupposée de la loi ou du texte de loi, mais bien sur l'interprétation qu'en génèrent le législateur et l'appliquant du travail du législateur, en l'occurrence le juge. Pour rester dans notre propos, l'analogie, ici, voudrait que le juge produise un jugement en citant le texte de loi, tout en gardant à ce dernier son intégrité juridique, au même titre que le critique universitaire produit un jugement de valeur en citant diverses sources d'autorité. Dans le cadre de la réflexion de Géa, la dimension d'extranéité du texte de loi et des circonstances de son interprétation confère dès lors sa validité à la décision judiciaire particulière, celle notamment du juge, laquelle à son tour peut être intégrée, ne serait-ce que dans le principe du droit français, à l'ensemble du système juridique sous la modalité de la jurisprudence :

Sans le savoir, Bakhtine introduit (ou introduisait) là une conception des plus pertinentes pour saisir le mode d'élaboration de la jurisprudence - ou, plus largement, le montage qui préside à la constitution des normes en certaines strates de la fabrique du droit. Cette conception offre, à notre sens, un prisme, que la théorie de l'interprétation gagne à intégrer dans son dispositif, et dont les fruits méritent d'être recueillis, pour qu'il en soit tiré toute la substance. «Jurisprudence » : ce terme désigne, sous notre plume, une part - une part seulement - de l'activité contentieuse qu'alimentent les litiges soumis aux tribunaux et dont procèdent les décisions judiciaires. Telle que nous l'envisageons, dans la lignée d'Antoine Jeammaud et Evelyne Serverin, la jurisprudence se présente comme une strate du droit positif - du droit en vigueur - composée d'un ensemble d'interprétations, d'énoncés normatifs, de qualifications, mobilisés, consacrés ou façonnés - implicitement ou explicitement - par des décisions dont la 
régularité ou l'autorité de la juridiction qui en est l'auteur permet de pronostiquer la réitération. Elle est, en somme, cette part de la masse des décisions de justice, d'où émanent, par une singulière alchimie, des interprétations normatives, des normes générales et abstraites. C'est à ce niveau, dans cette strate jurisprudentielle, que le dialogisme se déploie, à nos yeux, de façon éclatante - tout du moins en droit français. (279)

Pour nous, c'est à travers le même paradigme bakhtinien de l'exotopie que peut se comprendre la valeur assertive de la citation dans la pratique du critique littéraire universitaire. La citation, en maintenant sa valeur axiologique et en même temps son extranéité à l'argumentation du critique, permet à ce dernier de formuler les termes de son «jugement » sur les faits étudiés. Tout comme dans la pratique judiciaire, la citation, dans cette dimension nécessaire d'extranéité validante, ne saurait se suffire du résumé ou de l'évocation parcellaire à laquelle elle se ramène dans la pratique actuelle du discours critique universitaire, comme le manuel cité en début de propos le stipule. Si la citation, dans cette circonstance, se retrouve réduite à la mention ou à la reprise minimalisée du fait assertif qu'elle apporte, autrement dit, les réflexions existantes, une telle pratique remet en question la nécessité d'objectivité attendue du travail scientifique, y compris dans les sciences humaines. Ici, comme dans la pratique judicaire, et particulièrement à l'exemple du recours à la jurisprudence, qui fait penser à la démarche du critique universitaire dans son recours à différents travaux de recherche, eux-mêmes en concordance ou en divergence avec des théories ou perspectives herméneutiques existantes, la citation mérite d'être présentée tant dans son contexte d'intelligibilité que dans sa valeur argumentative d'origine. Un tel principe est au cœur du processus de prise de décision judiciaire :

En scrutant les raisonnements déployés par les magistrats de la Cour de cassation en amont de leurs décisions, dans la phase préparatoire du processus décisionnel, l'on s'aperçoit que les interprétations se forgent au contact d'une floraison de textes et de discours, de source et nature diverses (textes législatifs ou réglementaires, précédents jurisprudentiels, etc.), et, surtout, que le(s) sens attribué(s) au texte(s) interprété(s) se constitue(nt) en interaction vive et constante, pour reprendre la formule de Bakhtine, avec les interprétations qui ont pu lui être conférées en amont et celles que cette interprétation jurisprudentielle pourra, le cas échéant, engendrer lors de sa réception. La doctrine juridique, suivant la dénomination consacrée par le langage juridique français, c'est-à-dire la « communauté savante d'interprétation » (via les travaux et réflexions qu'elle produit, et les fonctions qu'elle assume), joue, à ce titre, un rôle central, fondamental. C'est, dans une large mesure, par le dialogue entre jurisprudence et doctrine tel qu'il se concrétise sur le registre de l'interdiscours, par ce dialogue interne, cette « dialogisation intérieure du discours » (Bakhtine, 1934/1975/1978: 
102-103), que le sens de la norme ou de l'interprétation jurisprudentielle se constitue. Hypothèse de la constitution dialogique du sens de la norme ou de l'interprétation jurisprudentielle. (279-280)

C'est un tel principe « dialogique » mais juridique de la construction du sens par l'individu juge ou critique universitaire, qui gouverne par exemple l'étude d'Alain Goldschläger sur l'œuvre de Simone Weil, ou dont l'absence explicite les problèmes épistémiques que pose un texte aussi fondamental que la Genèse biblique. Dans le cas de l'étude de Goldschläger, la mise en contexte des sources de l'étude ainsi que leur mention - citation - ultérieure dans la monographie constituée, font ainsi l'objet de l'introduction que le critique propose de sa monographie :

Être singulier et penseur hors du commun, Simone Weil nous a légué une œuvre présentant une difficulté particulière : elle n'a laissé en effet que des notes, des citations, des cahiers, des lettres, des réflexions éparses, des anecdotes mythologiques, des questions jetées sur papier, au jour le jour, au gré de ses préoccupations. Seuls les articles qu'elle publia de son vivant échappent à cet état d'inachèvement.

La philosophie de Simone Weil reste souvent énigmatique à cause de sa densité ; une étude des origines intellectuelles de la réflexion de la jeune femme ne peut qu'améliorer notre compréhension d'une œuvre qui se dérobe à nous par tant d'aspects. Il convient donc de nous pencher sur les sources de son inspiration que nous connaissons. $[\ldots](10)$

Pour la Genèse biblique, c'est plutôt l'absence d'indications des contextes et sources du texte qui constitue le nœud des contradictions inscrites à même ses références au discours divin, sans possibilité de vérification ou d'assertion, mais telles que ces contradictions auront servi de fondement à des modèles de société, à l'exemple des cultures occidentales aujourd'hui, ou de la « civilisation judéo-chrétienne » plus généralement, et telles que leurs contenus font l'objet de remise en cause également aujourd'hui, ne serait-ce que dans la perspective féministe, sur la question particulière de la femme à partir du mythe de la Chute (Lawson-Hellu).

Dans un cadre épistémologique de réflexion comme celui de la perspective postcoloniale appliquée à l'œuvre littéraire, cadre où la lecture de l'œuvre en fait un outil de résistance, même symbolique, c'est-à-dire une œuvre politique, la prise en compte de la nécessité de contextualisation et de maintien de l'intégrité des «appuis au texte » de l'étude devient un impératif qui excède la simple nécessité d'objectivité scientifique. Là, le volume de la citation ne dépend que de la pertinence argumentative de la source invoquée, c'est-à-dire de sa valeur 
d'« évidence » dans l'argumentation proposée. Le cas d'étude présenté ici, qui concerne la relecture postcoloniale de l'œuvre littéraire de Couchoro, en donne une mesure.

\section{Le cas d'étude}

Dans ce cas d'étude, il s'agit notamment de la réfutation des thèses colonialistes apposées à l'œuvre de cet écrivain, où l'argumentation qui fonde la réfutation, c'est-à-dire les résultats de recherche proposés (notre relecture postcoloniale de l'œuvre littéraire de l'écrivain), repose sur la citation de la thèse initialement présentée sur l'écrivain dans la spécificité de sa propre argumentation. Pour cette thèse initiale, il s'agit de l'analyse pionnière de l'œuvre de Couchoro par Alain Ricard, spécialiste de l'œuvre de cet écrivain, et c'est le volume élevé du recours à la citation dans la réfutation qui permet de suivre les termes de la relecture ainsi proposée, particulièrement sur la question de l'inscription de la tradition dans l'œuvre de l'écrivain.

Pour l'intelligibilité de l'œuvre de Couchoro dans son rapport à la question de la tradition posée par l'histoire coloniale, où il s'agissait pour le fait colonial de proposer des principes associés à la modernité en opposition à la tradition, c'est-à-dire aux valeurs locales, nous dirons que la résistance anticolonialiste de l'écrivain dans son œuvre comme dans son parcours intellectuel et politique ne saurait entériner la lecture critique proposée de cette œuvre. Une telle lecture critique y relève notamment un assujettissement à l'idéologie coloniale à travers les valeurs du christianisme et de la modernité qui, par ailleurs, accompagnent le discours colonial et informent l'œuvre de l'écrivain. Si l'écrivain ne souscrit pas à l'idéologie coloniale et à ses valeurs, l'inscription du fait religieux chrétien et du fait socioculturel «moderne » dans la pertinence sémiotique de son œuvre ne peut dès lors que se redéfinir.

Le champ littéraire francophone en Afrique s'est défini dans ses premières générations par la dualité tradition-modernité, suivant les termes idéologiques du discours colonial. Dans les termes de cette définition du champ institutionnel, l'œuvre de Couchoro s'inscrirait dans la «modernité ». C'est le sens de la lecture que propose de son œuvre Guy Ossito Midiohouan :

Les professions de foi chrétienne que Couchoro met dans la bouche de ses personnages, leur confiance absolue en Dieu, la condamnation des croyances et de l'usage des forces occultes (gris-gris, sorcellerie) s'expliquent dans une large mesure par son éducation : il fit ses études primaires à l'École catholique de GrandPopo, entra au Séminaire Sainte-Jeanne d'Arc de Ouidah où il passa deux ans (1915-1917) avant de devenir moniteur à l'École catholique de Grand-Popo (19171924). (70) 
Pour Couchoro, cependant, dont l'œuvre s'inscrit dans la tradition locale, tant dans sa forme, dans ses thèmes que dans son discours de résistance, il s'agit d'une tradition qui se reconnait dans la permanence de valeurs culturelles nécessaires à l'intégrité du groupe, et qui reconnaît aussi l'apport de l'extérieur pour autant que cet apport ne remette pas en cause l'intégrité du groupe. C'est dans cette perspective qu'il réécrit par exemple une de ses œuvres de la période coloniale, Amour de féticheuse (1939), dans l'après-indépendance, avec Amour de féticheuse au Togo (1966), en précisant qu'il s'agit, pour cette nouvelle version, d'une « nouvelle » œuvre où il met en scène les passions humaines et non les «pratiques animistes de chez nous », comme il l'indique : « Notre propos est de suivre le choc des passions humaines : aucune secte religieuse ne garde le monopole du Bien ou du Mal en tant qu'action humaine. Sur tous les bords se rencontrent des âmes justes comme des franches canailles » $(2,295)$.

Chez l'écrivain, il s'agit également d'une conception de la tradition qui correspond à la définition épistémique du principe de la culture, c'est-à-dire d'une conception de la tradition qui, en intégrant des faits de culture associés à l'étranger local ou européen, récuse le principe de la tabula rasa propre au fait colonial et défendu comme tel dans l'idéologie coloniale ainsi que dans le principe de la modernité. Si, déjà, dans un de ses premiers romans, Drame d'amour à Anecho (1950), il souligne par exemple l'excellence de l'anglais parlé par ses personnages, le propos pour lui demeure, en cela, la réponse qu'il appose au discours imagologique de l'idéologie coloniale qui avilit l'être « noir » colonisé :

Comme elle allait répondre, elle vit quelqu'un venir par la rue de Lagbonou. Une intuition, encore vague, l'envahit soudain. Elle se dégagea de l'étreinte du jeune homme.

Précipitamment, ils rectifièrent leur attitude. Elle reconnut celui qui venait.

- Eliot, fit-elle tout haut, est-ce encore toi ? Lui, fou de rage, dédaigna de répondre.

- Cher Monsieur excusez-moi : j'ai à vous parler, dit-il en anglais.

- Devant Mercy ? demanda froidement Kuanvi en anglais aussi.

- Oui, puisque la chose l'intéresse.

Ils étaient tous deux en bras de chemise... De passants, presque pas. $(1,283)$

Il en va de même du portrait qu'il propose, par exemple, de la femme mina, toujours de « chez nous », comme il l'indique dans le même roman, où ne se distinguent finalement dans la finesse qu'il prête à cette «femme mina », ni la « chrétienne », ni la «païenne » : 
Sur la place de Fantekome et du côté de la gare, des femmes minas parées, retardées sans doute par les apprêts de toilette, se hâtaient, les unes vers le temple, les autres vers l'église, car, de part et d'autre, l'office venait de commencer.

Le petit marché de la gare s'agitait déjà : les femmes païennes, indifférentes aux cérémonies du culte chrétien, circulaient et s'égosillaient autour des éventaires de crevettes, de poissons frais, de légumes et d'épices. Une sourde rumeur faite d'éclats de voix et de cris se mêlait alors à la note poétique que les grandes fêtes religieuses ne manquent jamais de donner, chaque année, aux jours populaires de chez nous. (236)

C'est d'un rapport à la tradition qu'il s'agit ainsi et qui s'inscrit en porte à faux à la lecture de son œuvre proposée par exemple dans les travaux de Ricard. Dans ce rapport, pour l'écrivain, la femme, en tant que mère, occupe notamment une place primordiale, si l'on concède que la femme demeure «gardienne de la tradition », nonobstant la position de la perspective féministe à l'encontre d'une telle assertion.

Dans le premier chapitre de son ouvrage consacré à l'écrivain, Ricard présente le rapport entre les spécificités discursives de l'œuvre littéraire de Couchoro et le parcours de l'écrivain à partir de la perspective coloniale de l'éducation de ce dernier (19-20). Pour le critique, cependant, c'est dans le rapport entre la tradition catholique de l'éducation de l'écrivain et le fait colonial français, ainsi que dans le rapport subséquent entre le fait colonial et son parcours intellectuel, que se situe la pertinence discursive de son œuvre :

Couchoro est né dans une famille catholique. Cela est important pour l'orientation de sa carrière politique : le catholicisme est français, après avoir été portugais sur cette côte. La France est la protectrice de l'Église de Rome et malgré l'anticléricalisme officiel, bien peu sera fait pour contrer l'effort d'éducation des élites entrepris par l'Église catholique. (20)

Pour le critique, également, c'est dans cette perspective coloniale française qu'il convient de situer les trois thèmes qu'il propose comme fondements à la pensée politique de l'écrivain, le régionalisme, le nationalisme et le socialisme :

Il nous paraît possible d'organiser tous les textes non romanesques, c'est-àdire aussi bien les reportages de L'éveil togo-dahoméen, que les essais politiques comme Les dix plaies de l'Afrique, autour des trois thèmes du régionalisme, du nationalisme et du socialisme. Où avons-nous trouvé ces étiquettes ? Félix Couchoro nous a lui-même fourni les deux dernières puisqu'il conclut son dernier essai politique par un appel au socialisme en 1967 et qu'il a été pendant près de 20 ans - de 1941 à 1960 -, associé aux entreprises des nationalistes togolais, en particulier par ses collaborations journalistiques. (20-21) 
Dans cette lecture qui en est proposée, le «régionalisme », dans la pensée politique de Couchoro, se définirait ainsi dans les termes coloniaux de l'inventaire des cultures locales :

La première étiquette est celle qui nous pose le problème le plus difficile. Le régionalisme est en effet dans le Dahomey des années vingt le programme de la revue Reconnaissance africaine fondée par le Père Aupiais, directeur du séminaire de Ouidah à partir de 1919, année où Félix Couchoro quitte précisément ce séminaire. [...]

Le rôle du Père Aupiais, missionnaire à Porto Novo dès 1903, a été analysé par Georges Hardy qui lui a consacré un ouvrage. [...] Félix Couchoro a donc dû être en contact, dès le début de sa carrière journalistique, avec un milieu ouvert à la promotion des valeurs de la culture africaine. (21-22)

Pour Ricard, qui retient l'exemple du premier roman de l'écrivain, L'Esclave (1929), et de la collaboration de l'écrivain à la revue La Dépêche africaine à Paris, c'est un régionalisme qui se définit en outre dans le cadre du projet assimilationniste du colonialisme français :

Il est important de saisir l'articulation entre régionalisme et universalisme pour comprendre la collaboration de Félix Couchoro - véritable début de sa carrière - à un journal, créé en France en 1928, La dépêche africaine [...]. Plus rien en effet qui rappelle dans ce journal la presse catholique missionnaire imprimée sous l'œil bienveillant de l'administration. La dépêche africaine émane du Comité de Défense de la Race Noire, une organisation issue d'une scission intervenue au Comité de Défense de la Race Nègre entre éléments nationalistes proches du Parti Communiste - qui devaient fonder une nouvelle organisation, la Ligue de Défense de la Race Nègre -, et des éléments plus modérés, partisans d'une politique non plus indépendantiste, mais assimilationniste, comme M. Satineau. [...]

Or L'esclave entre clairement dans les visées de La dépêche africaine puisque c'est un roman indigène " assimilé » à la littérature coloniale qui est ici présenté. Si le thème est banal - l'adultère -, le cadre tropical l'est moins, et surtout le drame est, cette fois, vécu de l'intérieur, puisqu'il est l'œuvre d'un indigène. L'exotisme ne se nourrit pas ici de bizarrerie ou de barbarie, mais de banalité ; il s'agit bien d'un exotisme " assimilé » et c'est là une première de la littérature coloniale puisque le livre de B. Diallo manque tout à fait de ce pittoresque dont Félix Couchoro semble avoir à revendre (marché, funérailles, nuit de noces, tamtam...). (22-24)

C'est dans le cadre de ce régionalisme assimilationniste colonial qu'il convient ensuite, pour le critique, de circonscrire le nationalisme dans la pensée politique de Couchoro, à partir, ici aussi, de l'exemple de L'esclave et de la collaboration de l'écrivain à La dépêche africaine :

Couchoro, dans une longue préface, explique sa démarche et en situe le cadre. Ce texte mal connu s'éclaire si on le situe dans la lignée « régionaliste » de Reconnaissance africaine et « assimilationniste » de La dépêche africaine. Le régionalisme de Félix Couchoro est d'abord la description d'un fleuve, le Mono, 
voie de communication et d'échange. Il en rappelle la prononciation exacte et énumère le nom des ethnies qui vivent autour du fleuve [...]. (26)

Si la relation intrinsèque entre l'espace culturel guin-ewe, qui s'étend notamment de part et d'autre de ce fleuve Mono situé aujourd'hui entre le Togo et le Bénin pour une grande part, si cette relation donc, pour clore le propos ici, et le militantisme politique de l'écrivain dans le cadre ultérieur du nationalisme ewe infirment foncièrement une telle lecture, c'est sur la base de ce nationalisme ewe que se comprend la posture résistante de l'écrivain telle qu'elle informe son œuvre romanesque, et tel que le reconnaît pourtant Ricard dans le parcours journalistique de l'écrivain, notamment dans les journaux qu'il crée successivement :

Installé à Grand-Popo où il est employé au comptoir de la SCOA, c'est vers le Togo que le portent ses intérêts. La place est libre en effet à l'ouest du Mono pour le journalisme [...]. À la fin de 1931 naît L'éveil togolais qui devient en mars 1933 L'éveil togo-dahoméen, directeur-gérant : Félix Couchoro. Le journal tire à 300 exemplaires et tiendra jusqu'en 1935. [...]

La création de L'éveil togo-dahoméen est bien une nouveauté. Elle est ainsi justifiée dans les colonnes du journal : «Quant au Togo, il n'avait aucune feuille locale ; nous avons préféré nous y installer afin qu'il ne soit pas dit que de toutes les colonies de l'ouest africain le Togo seul restait en minorité sous le rapport de la presse (numéro 15-16, mai-avril 1933). » [...]

Dans le cadre de la légalité française et d'une revendication assimilationniste qui était celle de La dépêche africaine, L'éveil togo-dahoméen garde le ton qui caractérise une bonne partie de cette presse dahoméenne : une aimable causticité, prompte à relever tous les abus de l'administration et à revendiquer, pour les Noirs, les droits des Blancs. Rien de révolutionnaire, seulement une revendication de justice ; les journaux voulaient se situer sur un terrain solide. (28-30).

L'impression de tentative d'édulcoration des faits rapportés dans les dernières phrases de la citation cache mal ce que l'on inscrit d'ordinaire sous le paradigme de la « résistance » et ici, de la résistance anticolonialiste, comme ce fut le cas, historiquement, dans le parcours intellectuel et littéraire de l'écrivain. Un résumé de l'argumentation réfutée, suivant la consigne du recours minimaliste à la citation, n'aurait pas permis de relever les incohérences ou les traces du propre discours colonial qui interfère avec l'analyse ainsi proposée de l'œuvre de l'écrivain.

\section{Ouvrages cités}

Angers, Maurice. Initiation pratique à la méthodologie des sciences humaines. Anjou : CEC. 1996. Couchoro, Félix. Euvres complètes, Tome 1. Romans. London, ON : Mestengo P, 2005. 
---. Euvres complètes, Tome 2. Romans et récits. London : Mestengo P, 2006.

Faye, Babacar. « Non-coïncidence du dire et mise en scène de l'hétérolinguisme dans les écritures francophones africaines ». Les cahiers du GRELCEF 2 (2011) : 145-165.

Géa, Frédéric. «Mikhaïl Bakhtine et la théorie du droit : une rencontre heuristique ». Dialogues with Bakhtinian Theory. Proceedings of the Thirteenth International Mikhaïl Bakhtin Conference. Dir. Mykola Polyuha, Clive Thomson et Anthony Wall. London : Mestengo P, 2012. 275-286.

Goldschläger, Alain. Simone Weil et Spinoza. Essai d'interprétation. Québec : Naaman, 1982.

Harlow, Barbara. Resistance Literature. New York : Methuen, 1987.

Kourouma, Ahmadou. En attendant le vote des bêtes sauvages. Paris : Seuil, 1998.

Lawson-Hellu, Laté. « De l'axiologisation du « Récit-Bible » à la question épistémique de la « micro-identité » ». Les cahiers du GRELCEF 4 (2013) : 107-140.

Midiohouan, Guy Ossito. L'idéologie dans la littérature négro-africaine d'expression française. Paris : Harmattan, 1986.

Moura, Jean-Marc. Littératures francophones et théorie postcoloniale. Paris : PUF, 1999.

Randall, Marilyn. «Critiques et plagiaires ». Christian Vandendorpe. Le plagiat. Ottawa : PU d'Ottawa, 1992. 91-104.

Ricard, Alain. Naissance du roman africain, Félix Couchoro (1900-1968). Paris : Présence africaine, 1987.

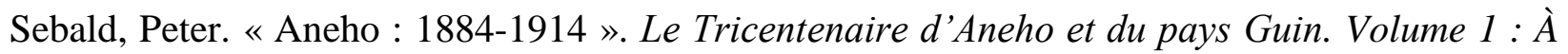
l'écoute de l'Histoire. Dir. N. L. Gayibor. Lomé : PU du Bénin, 2001. 235-276

Note

${ }^{1}$ Pour le critique, le principe de la non-cö̈ncidence $d u$ dire concerne l'inadéquation d'un terme à rendre compte d'une réalité donnée et, nécessitant de la part de l'énonciateur, le recours aux diverses modalités de l'explication ou du métapropos. 